\title{
REHABILITACIÓN NEUROPSICOLÓGICA EN PACIENTES CON TUMORES CEREBRALES
}

\author{
NEUROPSYCHOLOGICAL REHABILITATION PROGRAMS IN ADULT PATIENTS WITH \\ BRAIN TUMORS
}

\begin{abstract}
Ana Sanz Cortés ${ }^{7}$ y María Eugenia Olivares Crespo²
' Fundación Grupo IMO. Departamento de Psico-oncología y Unidad Funcional de Neuro-oncología

2 Servicio de Obstreticia y Ginecología. Hospital Clínico San Carlos. Facultad de Medicina. Universidad Complutense Madrid.
\end{abstract}

\section{Resumen}

Los déficits cognitivos son uno de los síntomas más frecuentes en pacientes con tumores cerebrales, principalmente en atención, memoria y funciones ejecutivas. Estas alteraciones impactan de forma negativa en al calidad de vida de los pacientes y en su reincorporación a su funcionamiento diario. En los últimos años se están desarrollando programas de Rehabilitación Neuropsicológica en pacientes adultos con tumores cerebrales, con resultados positivos. Así, los autores recomiendas que las intervenciones incluyan técnicas de psico-educación, estrategias de recuperación de los diferentes procesos atencionales y de ejecutivos (planificación y organización), así como técnicas compensatorias para la memoria. Los trabajos publicados hasta la fecha muestran mejoría inmediatamente después de la intervención en atención y memoria. Sin embargo, no existen datos del impacto de estos programas de Rehabilitación en el rendimiento de estos pacientes a medio y largo plazo (más de seis meses). Futuros estudios necesitan evaluar la eficacia de este tipo de intervención en pacientes mayores con tumores cerebrales, así como diferenciar los resultados en función del tipo de tumor y de los tratamientos oncológicos aplicados, ya que estas dos características pueden afectar de forma significativa a los datos obtenidos.

Palabras clave: tumores cerebrales, pacientes adultos, Rehabilitación Neuropsicológica.

\section{Abstract}

Cognitive deficits are one of the most common symptoms in patients with brain tumors, especially in attention, memory and executive functions. These changes impact negatively on the quality of life of patients and their return to their daily activities. In recent years, they are developing Neuropsychological Rehabilitation programs in adult patients with brain tumors, with positive results. The authors recommend that interventions include psychoeducation techniques, recovery strategies of different attentional and executive processes (planning and organization) and compensatory techniques for memory. The studies published to date show improvement immediately after surgery in attention and memory. However, there are not data exist on the impact of these programs on performance rehabilitation of these patients in the medium and long term (over six months). The future studies need to evaluate the effectiveness of this intervention in elderly patients with brain tumors, and the results differ depending on the type of tumor and cancer treatments applied, as these two features can significantly affect the data obtained.

Key words: brain tumors, adults, neuropsychological rehabilitation.

\section{Correspondencia:}




\section{INTRODUCCIÓN}

Para la elaboración de este artículo se ha realizado una revisión bibliográfica en las bases de datos de Pubmed y EMBASE, con las palabras clave effective cognitive rehabilitation and brain tumors. Además, se ha consultado en Cochrane library la existencia de evidencia científica al respecto, en las revistas de acceso libre de Biomed y en tratados sobre Neuro-rehabilitación. Los aspectos específicos del desarrollo evolutivo de la población infantil, así como las características diferenciales de los tumores cerebrales, de los síntomas asociados, de la planificación y del contenido de los programas de rehabilitación entre la esta población y la adulta, obligan a abordar la eficacia de estas intervenciones de forma separada. Por ello, en la presente revisión se expondrá información específica sobre las estrategias de rehabilitación neuropsicológica en pacientes adultos con tumores cerebrales, primarios o metástasis.

En este sentido, el término de tumor cerebral abarca un amplio número de procesos neoplásicos con diferencias histológicas, incluidas las metástasis de tumores sistémicos ${ }^{(1)}$, que representan un total del $2 \%$ de todas las patologías oncológicas ${ }^{(2)}$. Una de las manifestaciones clínicas más frecuentes en estos pacientes son los déficits cognitivos, que se asocian a su vez a elevada morbilidad y a una negativa en su calidad de vida ${ }^{(3-5)}$, los que convierte a los tumores cerebrales en una de las principales patologías neurológicas con este tipo de sintomatología ${ }^{(6)}$.

Sin embargo, los datos de prevalencia sobre los déficits cognitivos en pacientes con tumores cerebrales son muy dispersos en función de los diferentes estudios, oscilando los porcentajes entre el $12 \%$ y el $90 \%$, durante todo el proceso de la enfermedad $^{(7-17)}$. En lo que sí parece haber acuerdo en la literatura, es respecto a los dominios cognitivos que presentan mayor afectación en estos enfermos ${ }^{(16,18-23)}$, entre los que cabe destacar:

- Atención: velocidad de procesamiento de la información y atención alternante.

- Memoria verbal: fijación, almacenamiento y/o recuperación de la información verbal

- Funciones ejecutivas: fluidez y generación de alternativas, memoria de trabajo y planificación, y por último, organización.

- Lenguaje: fluidez verbal y denominación verbal. Al contrario que otros procesos cognitivos, la presencia de alteraciones en esta función depende de la localización del tumor en el hemisferio dominante.

Además, se han diferenciado dos tipos de patrones sobre estas alteraciones y que pueden estar presentes a lo largo de la enfermedad ${ }^{(8,16,19)}$ :

a. daño cognitivo global o difuso, de gravedad leve o moderada, que cursa con un amplio número de síntomas asociados al efecto de masa de la lesión, a una rápida velocidad del crecimiento del tumor y a la presencia de crisis epilépticas;

b. déficit cognitivo específico relacionados con la localización anatómica de la lesión y que suele estar más presente en pacientes con gliomas de bajo grado.

De esta forma, la alta frecuencia de estos déficits, el aumento en las tasas de supervivencia de estos pacientes, junto con los avances tecnológicos en los tratamientos oncológicos han provocado un aumento del interés en las terapias de soporte y en los programas de rehabilitación en esta población.

El control de los síntomas neurológicos y la recuperación de los déficits cognitivos se asocia con una mejora en la calidad de vida ${ }^{(24)}$, objetivo a perseguir en el abordaje multidisciplinar de 
los pacientes oncológicos. Además, en aquellos enfermos con mejor pronóstico (neoplasias benignas o gliomas de bajo grado), este tipo de intervenciones facilita la reincorporación a las actividades en el ámbito familiar, social y laboral y, así poder reestablecer el funcionamiento previo. Dentro del ámbito de la Rehabilitación, los resultados actuales demuestran que las intervenciones neuropsicológicas son efectivas para la recuperación de los déficits cognitivos, principalmente los relativos a la atención y algunas funciones ejecutivas, así como la disminución de las alteraciones emocionales y conductuales presentes en pacientes con tumores cerebrales $^{(25)}$. Las estrategias y técnicas utilizadas en estos programas de rehabilitación se consideran como una práctica estándar o una opción práctica y se incluyen en las guías clínicas ${ }^{(25,26)}$.

A continuación se describe brevemente en qué consiste la rehabilitación neuropsicológica y los principios básicos de los programas de intervención.

\section{DEFINICIÓN DE REHABILITACIÓN NEUROPSICOLÓGICA}

La Rehabilitación Neuropsicológica se define como la aplicación de las estrategias de intervención o técnicas que tengan como objetivo permitir a pacientes $y$ familiares manejar, sobrellevar, o reducir el déficit cognitivo que se produce por una lesión cerebral(27). Así, este tratamiento supone la combinación de estrategias de intervención cognitiva y sistemas compensatorios, con métodos de intervención conductual y otras estrategias, destinadas a reducir los problemas emocionales y favorecer la integración social y laboral. El objetivo final de cualquier programa de Rehabilitación Neuropsicológica incluye el aumento de las capacidades funcionales del individuo, además de: a) mejorar el rendimiento de las funciones cognitivas; b) lograr mejoras funcionales mediante técnicas específicas; c) proveer entornos óptimos y adaptaciones ambientales; d) entrenar en estrategias compensatorias, y e) educar a la familia.

\section{Principios de los programas de Rehabilitación Neuropsicológica}

Para que un programa de intervención neuropsicológica sea considerado como eficaz debe cumplir unos principios básicos, de forma independiente a la causa del daño cerebral y al momento de la enfermedad en que se realice la rehabilitación ${ }^{(27,28)}$. Estos principios son:

1. Necesidad de un marco teórico consistente que asegure las bases científicas para establecer las jerarquías de los objetivos de tratamiento y el proceso terapéutico sistemático que organiza las actividades.

2. Organización jerárquica de las tareas respecto a su nivel de dificultad y al orden de prioridades. Tirapu y Muñoz ${ }^{(28)}$ consideran que es imprescindible establecer la planificación sobre las funciones cognitivas y los problemas emocionales sobre los que se va a trabajar y el orden de los mismos.

3. Perspectiva de trabajo interdisciplinar, ya que las alteraciones cognitivas, emocionales y físicas que se producen después del daño cerebral son muy complejas y, por lo tanto, no pueden ser abordadas de forma exclusiva por un único profesional. Desde estos programas, se aboga a la participación conjunta y coordinada en equipo.

4. Iniciar del programa de rehabilitación en las fases iniciales del daño cerebral. Se considera que la intervención desde los primeros momentos posteriores al daño incrementa las posibilidades de recuperación funcional, al maximizar los avances cognitivos que se producen en el periodo de recuperación espon- 
tánea. En pacientes con tumores cerebrales, este principio debe considerarse con precaución ya que no sólo el tumor es el responsable de los déficits cogniti$\operatorname{vos}^{(16)}$. La aplicación de los tratamientos oncológicos, la presencia de depresión clínica y otros factores se asocian con alternaciones cognitivas que en los momentos iniciales de la enfermedad no se evidenciaban ${ }^{(15,16,19,20,29)}$.

5. Disponer del tiempo suficiente de tratamiento para establecer los nuevos aprendizajes, consolidar las habilidades entrenadas y generalizar su empleo a las situaciones cotidianas. En la programación de estas intervenciones debe considerarse las dificultades en la velocidad del procesamiento de la información, muy frecuentes en pacientes con tumores cerebrales; ya que ésta supone un requisito esencial para el establecimiento de cualquier aprendizaje, lo que implica tiempo y recursos para conseguir un resultado satisfactorio.

6. Habilidades conservadas como base de los programas de rehabilitación. Desde esta perspectiva es necesario conocer los procesos que conservan las personas con daño cerebral, ya que en los casos que no pueda repararse una función cognitiva se planteará la sustitución o compensación de la misma. Así, este principio lleva asociado la realización de evaluaciones neuropsicológicas rigurosas que proporcionen esta información.

7. La rehabilitación debe centrarse hacia objetivos funcionales, es decir, cubrir las necesidades de autocuidado, independencia e integración social y laboral.

8. La consideración de las variables emocionales es imprescindible en la planificación de los programas de rehabilitación, que pueden verse afectadas de forma directa, por el daño orgánico o indirecta, como respuesta a la pérdida del funcionamiento cognitivo.

\section{Tipos de intervención neuropsicológica en pacientes con tumores cerebrales}

En un reciente estudio de revisión, Gehring et al. ${ }^{(6)}$ recogieron los tipos de técnicas e intervenciones más utilizadas en esta población, basadas en la evidencia científica disponible ${ }^{(25,26)}$, que deben aplicarse de forma combinada e incluir estrategias metacognitivas que faciliten la auto-regulación emocional y la generalización de su efecto a la vida diaria:

- Programas psicoeducativos. La característica principal de este tipo de intervención es que aportan información sobre el funcionamiento cognitivo, los déficits más frecuentes y sus consecuencias en la vida diaria, tanto de los pacientes como de los familiares. Además se incluyen habilidades generales de estimulación de la atención y la memoria.

- Entrenamiento directo sobre la función cognitiva afectada. Las estrategias generales de rehabilitación se aplican mediante la repetición de ejercicios y tareas $^{(30)}$, bajo el supuesto de que la red neural implicada en su ejecución se entrena y facilita el crecimiento axonal o la estimulación de vías aferentes ${ }^{(26)}$. Éstas se resumen en:

- Restauración: se centra en la recuperación de la función a través del entrenamiento directo sobre el proceso disfuncional.

- Compensación: supone la reorganización de procesos, fomentando que las capacidades preservadas se encarguen de minimizar la repercusión de los déficits.

- Sustitución: se relaciona con una nueva forma de afrontar los daños cuando las dos técnicas anteriores no son posibles, enseñando al paciente a emplear señales y dispositivos externos para minimizar las limitaciones. Actualmente el uso de programas de ordenador y nuevas tecnologías 
para el re-entrenamiento de determinadas funciones cognitivas se está convirtiendo en la modalidad de intervención elegida en pacientes con daño cerebral. Como se desarrollará más adelante, el equipo de Gehring et al. ${ }^{(31)}$ encontraron que, la rehabilitación mediante un software de simulación de conducción junto con un programa psicoeducativo se asociaban con una mejoría en el rendimiento de los procesos atencionales y de la calidad de vida en pacientes jóvenes con gliomas de bajo grado.

- Adquisición de estrategias internas. Estas técnicas están encaminadas a facilitar el afrontamiento y la adaptación de los déficits cognitivos asociados al tumor y a los tratamientos. Entre ellas se recogen: a) control y manejo de los estímulos distractores; b) planificación de actividades de forma eficaz; c) reglas de mnemotécnicas que faciliten los procesos de aprendizaje, y por último, d) solución de problemas y toma de decisiones.

- Estrategias del entorno. Se refiere a aquellas técnicas encaminadas a reestructurar el entorno del paciente para satisfacer las nuevas demandas de su actividad diaria ${ }^{(32)}$. Estas modificaciones externas deberán realizarse en función del tipo y del grado de severidad del daño cognitivo, siendo más necesarias cuanto más severo sea el mismo ya que estos casos suelen asociarse con un mayor pérdida de independencia.

\section{PROGRAMAS DE INTERVENCIÓN NEUROPSICOLÓGICA EN PACIENTES CON TUMORES CEREBRALES}

La literatura sobre la Rehabilitación Neuropsicológica en pacientes con tumores cerebrales es escasa, ya que hasta el año 2007 no se han realizado los primeros estudios prospectivos y ensayo clínicos que determinen la eficacia de este tipo de intervenciones en el rendimiento de diversos procesos cognitivos (ver tabla 1). De acuerdo a las recomendaciones de Cicerone et al. ${ }^{(26)}$, el denominador común entre varios estudios es la aplicación de estas intervenciones junto con técnicas cognitivo-conductuales y metacognitivas, para facilitar el afrontamiento adaptativo de la enfermedad, reducir y manejar los síntomas clínicos emociona$\operatorname{les}^{(6,33-35)}$. Las estrategias metacognitivas se refiere a aquellas técnicas que facilitan el aprendizaje de forma consciente de procesos y que permiten interiorizar conocimientos, también incluyen las acciones concretas que se realizan para mejorar el aprendizaje.

Por otra parte, el grupo de investigación de Gehring et al. ${ }^{(31)}$ han mostrado algunos factores, principalmente sociodemográficos, que se asocian con el éxito de este tipo de intervenciones y que ayudan a identificar aquellos pacientes que puedan beneficiarse de la rehabilitación neuropsicológica.

Previamente a esta fecha, Sherer et al. (36) publicaron una investigación de naturaleza retrospectiva que analizaba el efecto de la intervención cognitiva en 13 pacientes con tumores cerebrales malignos, que se aplicó seis meses después de la exéresis quirúrgica de la lesión. La rehabilitación neuropsicológica consistió en un programa con formato individual y grupal, de cinco horas semanales en las que se entrenaba en estrategias compensatorias de los déficits cognitivos, principalmente los relativos a los procesos de memoria. Los resultados, recogidos mediante una entrevista clínica posterior, mostraron que más de la mitad de los pacientes referían mejoría en las medidas funcionales y en sus actividades de la vida diaria. 


\begin{tabular}{|c|c|c|c|}
\hline 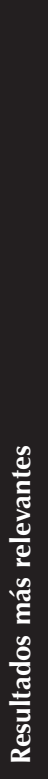 & 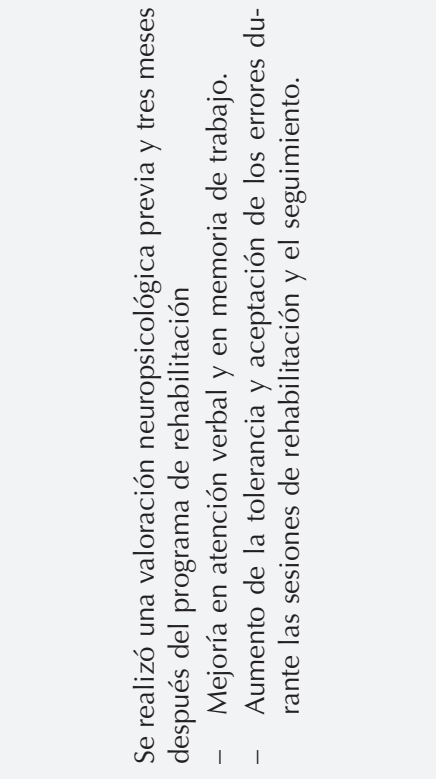 & 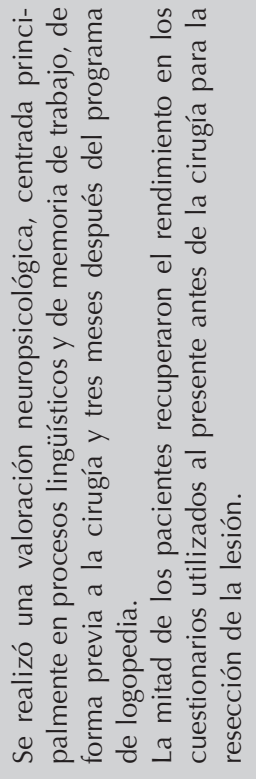 & 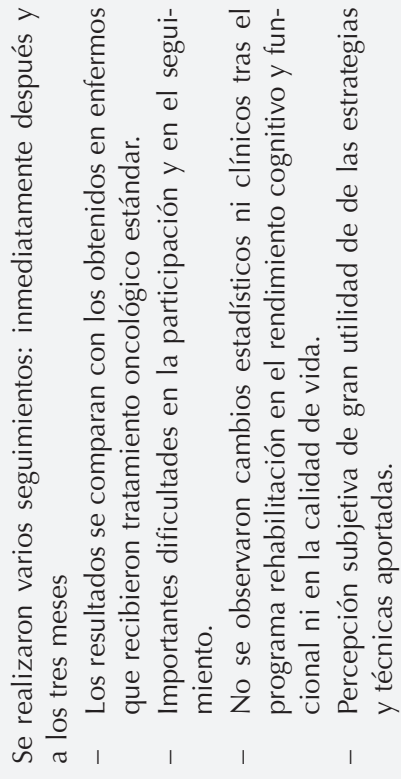 \\
\hline 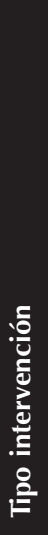 & 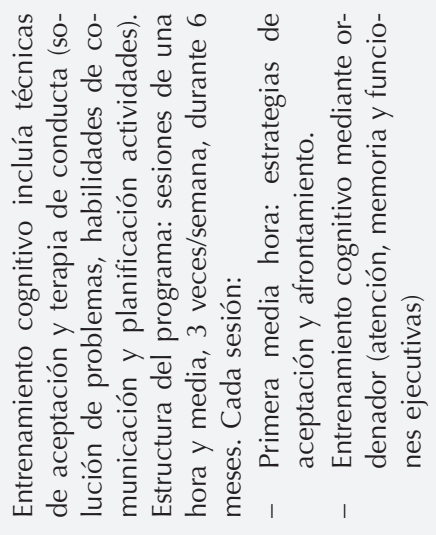 & 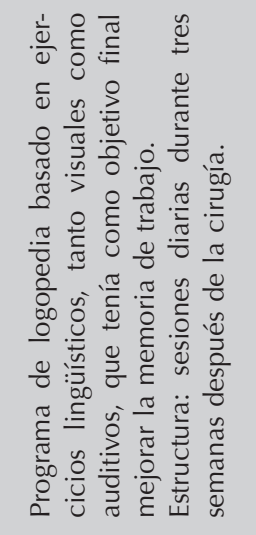 & 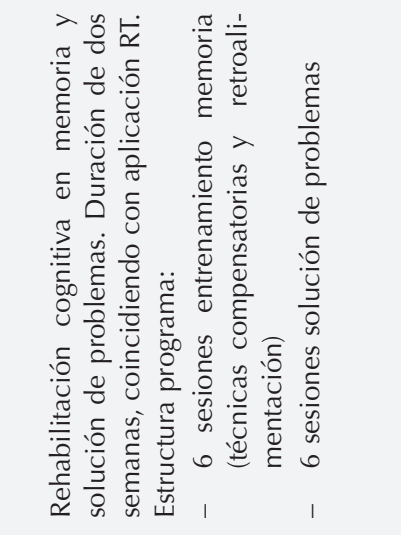 \\
\hline $\begin{array}{l}\frac{0}{0} \\
\frac{0}{0} \\
\frac{\pi}{0} \\
\frac{0}{0}\end{array}$ & 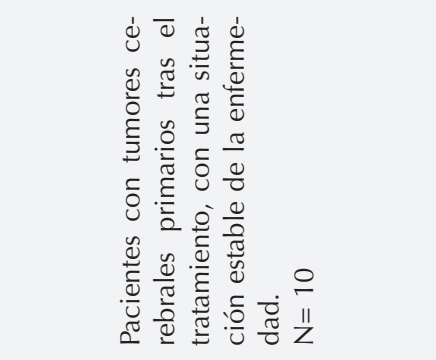 & 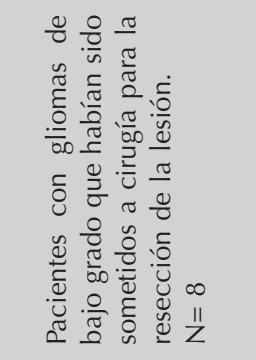 & 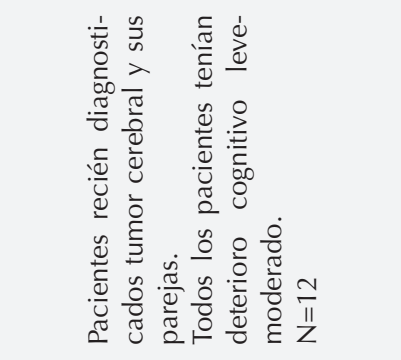 \\
\hline & 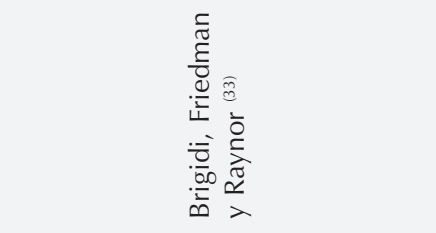 & 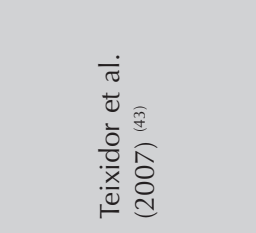 & 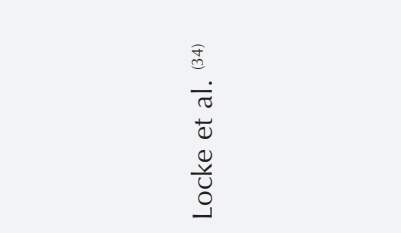 \\
\hline
\end{tabular}




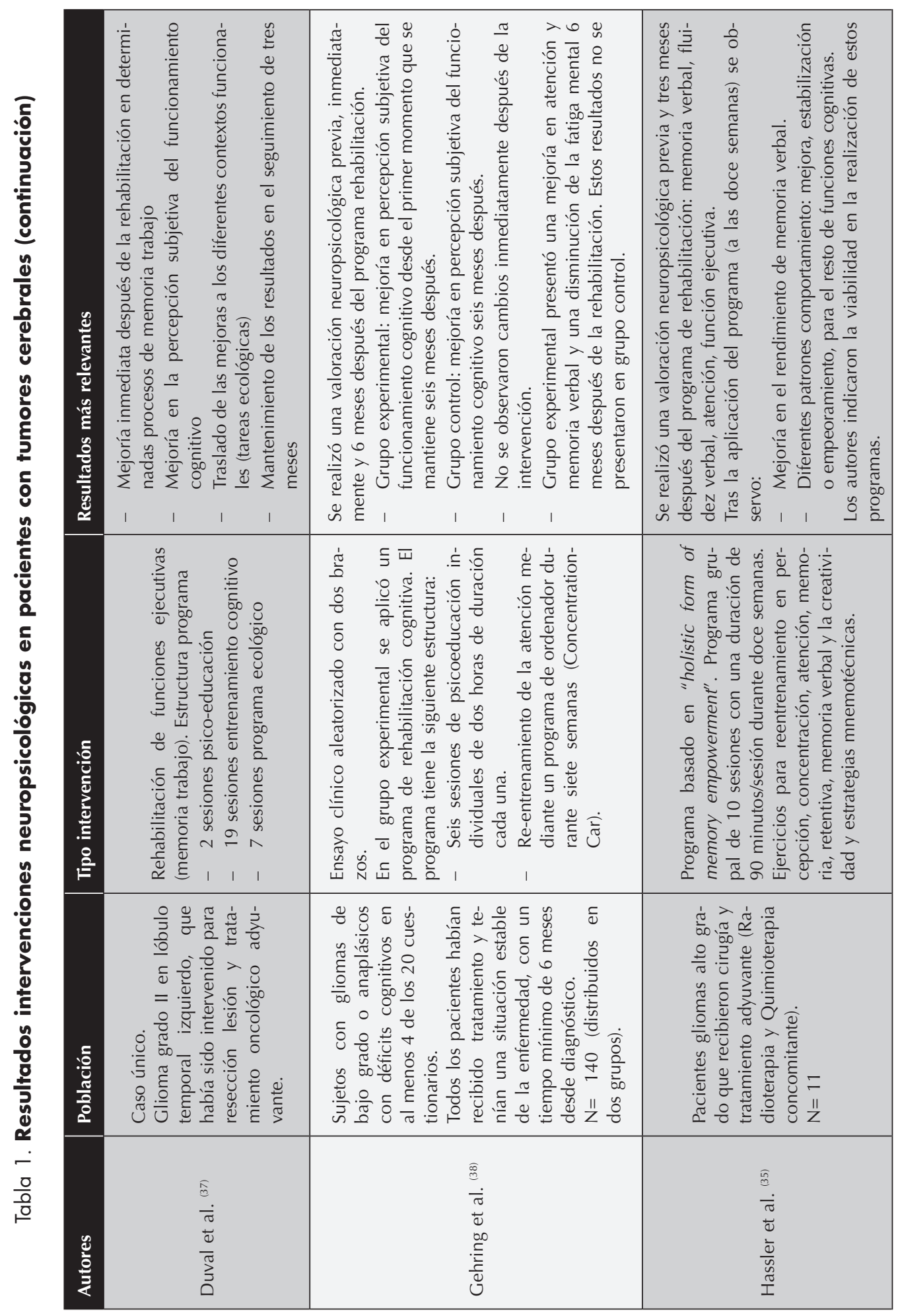




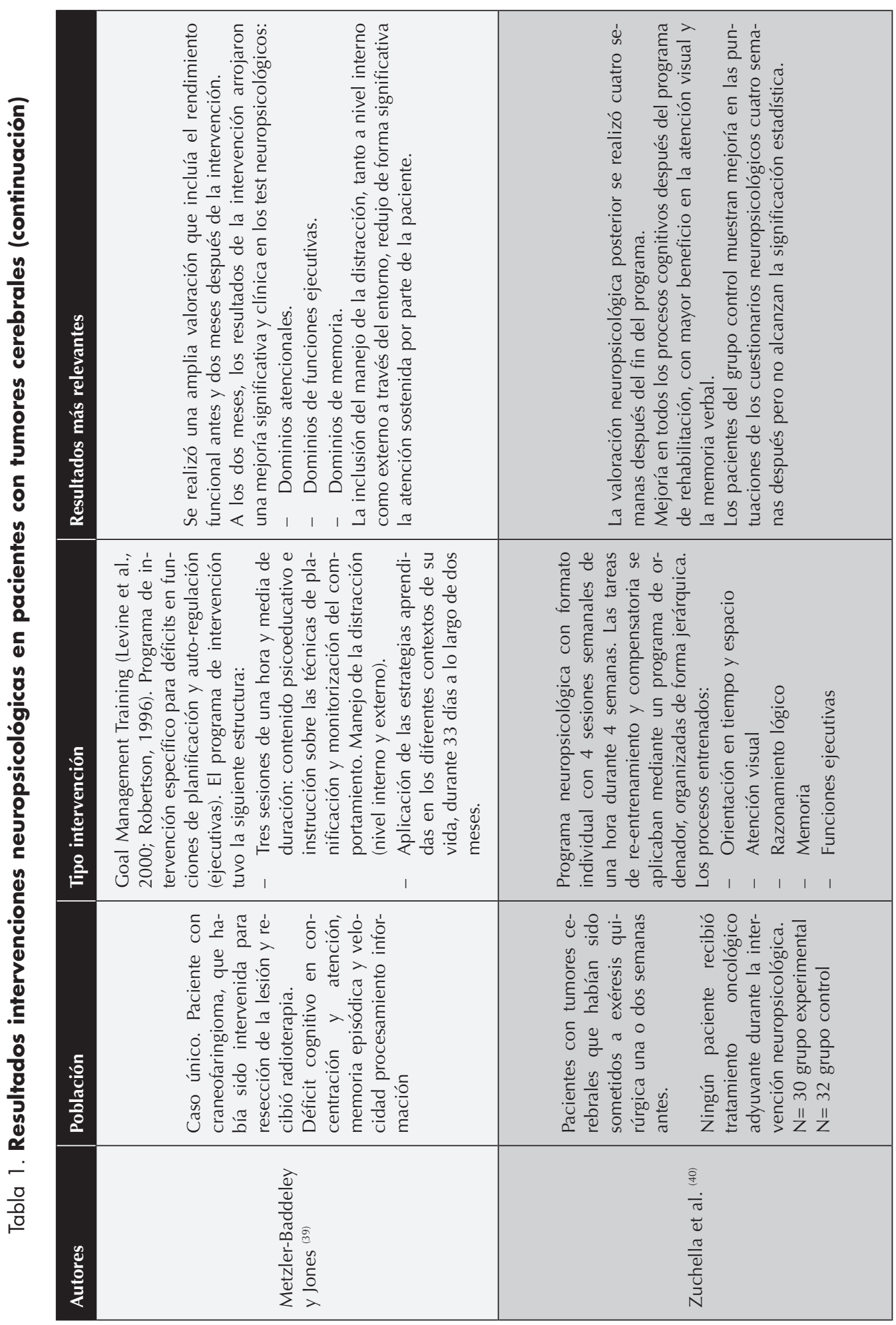




\section{Rehabilitación de procesos atencionales}

Como puede observarse en la Tabla 2, en la mayor parte de estudios hallados ${ }^{(31,33,34 \text {, }}$ ${ }^{37-40)}$ se utilizaron estrategias para la recuperación y compensación de los déficits en los diferentes procesos atencionales, al ser una de las funciones cognitivas más afectada desde los primeros momentos de la enfermedad e independientemente de la localización de la lesión ${ }^{(22)}$. La intervención de los déficits en la atención constituye un objetivo prioritario en las fases iniciales de los programas de rehabilitación, puesto que sólo en la medida en que se mejoren estas habilidades se podrán ir incorporando de manera progresiva componentes más complejos.

El programa de rehabilitación de la atención desarrollado por Gehring et al. ${ }^{(31,38)}$ está basado en el modelo clínico de la atención de Sohlberg y Mateer ${ }^{(41)}$ y en el entrenamiento Attention Process Training (APT) (42), propuesto por los mismos autores que se desarrollará más adelante debido a la relevancia que tiene en la práctica clínica. Además, estos investigadores ${ }^{(31,}{ }^{38)}$ complementaban la intervención con seis sesiones psicoeducativas acerca de la importancia de las funciones cognitivas en la vida diaria, las implicaciones de los déficits en su vida diaria y estrategias de estimulación de memoria y funciones ejecutivas. Tanto Gehring et al. ${ }^{(31,38)}$ como otros investigadores ${ }^{(33,37,39,40)}$ que incluyeron estrategias de recuperación sobre los diferentes procesos atencionales verbales y visuales, observaron mejoras clínicas al finalizar la rehabilitación y meses después de la misma. Además, se indicó que los pacientes aplicaban las habilidades aprendidas en su funcionamiento personal y se asociaba con una mejoría en la percepción subjetiva a nivel cognitivo ${ }^{(34,37,39)}$.

\section{Rehabilitación de funciones ejecutivas}

Las funciones ejecutivas y dentro de ellas, la capacidad de planificación y orga- nización así como la memoria de trabajo, han sido otros de los dominios sobre los que más se ha intervenido en pacientes con tumores cerebrales ${ }^{(31,3,37-40,43)}$ (ver tabla 3). En las investigaciones revisadas se han utilizado algunos de los componentes o el programa integral Goal Management Training(44) para mejorar las habilidades de planificación de estos pacientes, con resultados positivos sobre la eficacia del mismo. Éste se basa en la teoría de desorganización del comportamiento que propone que cada conjunto de comportamientos requiere unos objetivos que permiten crear una estructura de acciones y operaciones mentales para lograr dichas metas ${ }^{(45)}$. Así, al paciente se le enseña a controlar y monitorizar su conducta a través de los siguientes pasos: a) detener la actividad actual a tomar conciencia de la situación actual y orientar a la tarea; b) definir el objetivo principal y sub-objetivos de los componentes y comparar el estado actual con estos objetivos; c) ensayar los pasos necesarios para resolver el problema, y d) supervisar el resultado.

Respecto a la memoria de trabajo, Teixidor et al. ${ }^{(43)}$ analizaron los resultados de un programa específico de logopedia sobre el rendimiento de esta función, en pacientes con gliomas de bajo grado que habían sido sometidos a cirugía para la resección de la lesión. Los autores observaron tras la rehabilitación que más de la mitad de los individuos mostraban un rendimiento en los cuestionarios neuropsicológicos similar al presentado en los momentos previos a la exéresis quirúrgica. Por su parte, otros investigadores $^{(33,37)}$ indicaron que la aplicación de estrategias de recuperación y técnicas compensatorias se asociaba con mejoría en esta función meses después de la intervención para pacientes con tumores cerebrales después de los tratamientos y en ausencia de enfermedad.

Aunque no se considera una función ejecutiva, la velocidad de procesamiento 
de la información es un proceso fundamental para su correcto rendimiento, que suele estar afectado con bastante frecuencia en pacientes con tumores cerebrales. Por este motivo, algunos autores ${ }^{(31,33)}$ han considerado la necesidad de intervenir sobre este proceso mediante la aplicación del programa Time preassure managment $t^{(46)}$ que tiene como objetivo enseñar al paciente a manejar la presión del tiempo en la ejecución de las tareas y planificar dichas tareas con antelación, considerando el déficit en este proceso. Tanto en la intervención de la velocidad del procesamiento de la información como en la relativa a la memoria de trabajo, se considera necesario incluir entrenamiento en autoinstrucciones internas que ayuden a afianzar la adquisición de nuevos comportamientos.

\section{Rehabilitación de la memoria}

Los procesos de memoria han sido otros de los procesos cognitivos sobre los que más se han intervenido en pacientes con tumores cerebrales, observándose una mejoría clínica en los dominios verbales en la mayor parte de los estudios ${ }^{(35,38-40)}$. Estos resultados pueden deberse a la recuperación del funcionamiento en algunos niveles atencionales o por el trabajo directo en procesos mnésicos, como en el estudio de Hassler et al. ${ }^{(35)}$ que aplicaron un programa específico de memoria que incluía técnicas de mejoría de la concentración, entrenamiento en retención de material verbal y estrategias de mnemotecnia, para pacientes con gliomas de alto grado (ver tabla 1). En este sentido, Ciccerone et al. ${ }^{(26)}$ indicaron que las técnicas de compensación y el entrenamiento en técnicas de aprendizaje sin errores son las que mayor eficacia han mostrado en la rehabilitación de la memoria, ya que el paciente mediante estas estrategias reorganizan los circuitos neuronales intactos que se hacen cargo de parte de dichos procesos. Así, estas es- trategias pueden resumirse en: a) adaptaciones del entorno y ayudas externas; b) estrategias mnemotécnicas, y c) utilización de sistemas de memoria preservados.

Se considera relevante describir de forma un poco más exhaustiva una reciente publicación ${ }^{(40)}$, puesto que reúne dos características diferenciales con respecto al resto de estudios: el amplio número de procesos cognitivos que se incluyen en la rehabilitación neuropsicológica y el momento de aplicación de la misma, ya que se realiza de forma inmediata a la resección quirúrgica del tumor y antes de la aplicación de tratamientos oncológicos adyuvantes (entre una y dos semanas después de la misma). Los componentes del programa, que se entrenan mediante ejercicios y puzzles presentados por ordenador, son:

- Orientación temporal y espacial.

- Atención visual.

- Razonamiento lógico y cálculo.

- Memoria y reconocimiento.

- Funciones ejecutivas que incluían estrategias de autorregulación emocional, comportamiento social y juicio crítico, al igual que otras investigaciones.

El programa incluía una parte de comprensión y feedback de los ejercicios al finalizar las sesiones de rehabilitación para facilitar su generalización a los contextos de la vida diaria. Los resultados más relevantes referidos por los autores mostraron que cuatro semanas después de la finalización del programa se observó una mejoría de forma específica en procesos de atención visual y memoria verbal, con respecto a aquellos pacientes que no recibieron tratamiento. Asimismo, no se evidenciaron diferencias estadísticas ni clínicas en el rendimiento de las funciones ejecutivas ni respecto al uso de las estrategias metacognitivas.

En último lugar, algunos investigadores $^{(31)}$ incluyeron en los programas de rehabilitación neuropsicológica estrategias 
para manejar y disminuir la fatiga mental presenta en estos pacientes, y que puede tener un impacto directo sobre los déficits cognitivos. Los objetivos perseguidos en estos estudios son:

- En la planificación de las actividades se incluyen tiempos de descanso y mayor duración en la ejecución de las mismas para evitar este fenómeno.

- Mejoría en el rendimiento neuropsicológico que produce una menor fatiga mental, ya que requiere el consumo de un menor número de recursos cognitivos.

\section{Factores asociados a la eficacia de los programas de rehabilitación neuropsicológica}

Existen pocos estudios que determinen los factores asociados a la recuperación de los déficits cognitivos tras la intervención en pacientes con tumores cerebrales. Entre todas las variables consideradas, las sociodemográficas se definen como las que mejor predicen la recuperación del funcionamiento neuropsicológico en estos enfermos, al igual que ocurre en otras poblaciones con daño cerebral adquirido ${ }^{(47,48)}$.

En este sentido, los individuos de menor edad y con mayor nivel educativo se aprovechan de forma más satisfactoria y eficaz de las intervenciones neuropsicológicas ${ }^{(31)}$. Estos hallazgos parecen ser compatibles con la teoría de la reserva cognitiva, descrita en otras enfermedades del Sistema Nervioso Central, que suponen la existencia de un mecanismo activo que retrasa la neurodegeneración asociada a las mismas. Dicha reserva se basaría en la aplicación de los recursos aprendidos gracias a variables como el alto nivel educativo, profesión con exigencias cognitivas elevadas, actividades de ocio concretas, etc. ${ }^{(49)}$.

En el estudio de Gehirng et al.(31), se diferencian dos patrones de cambio diferentes respecto a la edad y la educación en las mejorías cognitivas tras el pro- grama de rehabilitación. Así, la variable edad fue la que mejor predijo la mejoría experimentada tras el programa de rehabilitación cognitiva (atención, memoria y funciones ejecutivas), y no se observó su efecto en la recuperación espontánea de los individuos que no recibieron intervención neuropsicológica. Respecto a la educación, un mayor nivel de estudios favorecía la recuperación de los déficits cognitivos independientemente si los pacientes recibían rehabilitación cognitiva o no, aspecto relacionado con el efecto de la práctica. Para aumentar la eficacia de los programas de rehabilitación cognitiva en pacientes de mayor edad, se considera necesario adaptar las características de los programas de rehabilitación al estilo funcional, vital y el nivel cognitivo de estos individuos. Para ello se tendrá que modificar el diseño y la planificación de las sesiones, el material que se utilizará y la forma de manejarlo.

\section{PROPUESTAS DE PROGRAMAS REPRESENTATIVOS DE INTERVENCIÓN NEUROPSICOLÓGICA}

A continuación se exponen de forma detallada dos tipos de intervención, la primera de ellas el Attention Process Training ya que es la base científica y teórica de otros programas de rehabilitación de la atención en pacientes con daño cerebral, independientemente de la etiología del mismo. El segundo programa que se describe es el creado por el equipo de Gehring et al. ${ }^{(31,38)}$ específicamente para pacientes con tumores cerebrales, que ha demostrado eficacia en la recuperación de déficits atencionales y de memoria.

\section{Programa ATP- Attention process training ${ }^{(42)}$}

Dicho programa combina métodos y técnicas utilizadas en rehabilitación cog- 
nitiva, psicología clínica y educativa, y está basado en el modelo conceptual de la atención de Sohlberg y Mateer ${ }^{(41)}$ que plantea cinco niveles clínicos: a) atención focalizada; b) atención sostenida; c) atención selectiva; d) atención alternante, y e) atención dividida.

El programa está estructurado como un conjunto de tareas ordenadas de forma jerárquica por su nivel de dificultad, aumentando de complejidad a medida que se consiguen los objetivos planteados y de este modo abordar todos los niveles de atención. Así, es aconsejable comenzar con aquellas que exijan demandas mínimas atencionales cuya realización requiera poco esfuerzo y así tener éxito en ellas, y posteriormente aumentar la dificultad de forma progresiva según se vaya alcanzando el criterio adecuado ${ }^{(50)}$. La aplicación del entrenamiento tiene un formato individual para que se adapte a los déficits de cada paciente y las necesidades de cada individuo, aprovechando su potencial y utilidad para la vida diaria de la persona.

En las últimas revisiones del programa se incluyen actividades de la vida diaria problemáticas para el paciente y se incluye el entrenamiento de otros elementos más complejos de control atencional y de memoria operativa. En la Tabla 2 se incluyen el tipo de tareas que se trabaja con cada dominio atencional y que se dividen en: a) tareas de cancelación visual y auditiva (cintas de audio); b) ejercicios de control mental, mediante el manejo de la información mentalmente, y c) tareas de la vida diaria. En cada sesión se emplean ejercicios de los diversos tipos de tareas y el orden de aplicación se deja a juicio del neuropsicólogo, siendo el formato de estas el siguiente:

- Explicación del nivel atencional que se va a trabajar, cuáles son los problemas que tiene cuando la atención no funciona correctamente.

- Práctica repetida de la tarea del programa; siendo cada ejercicio repetido hasta que se realiza correctamente tres veces consecutivas. Un ejercicio se considera adecuado cuando se hace en menos de cinco minutos o no se cometa más de cinco errores.

Para facilitar la generalización de lo aprendido a otros contextos, se recomienda el uso de autoinstrucciones, que así ponga en práctica en otras situaciones las habilidades que se van aprendiendo durante las sesiones ${ }^{(50)}$. Asimismo, estos autores indicaron la necesidad de entrenar al individuo en varios tipos de tareas (visuales, auditivas y táctiles) que exijan distintas respuestas, como hablar, apretar una tecla o escribir y conseguir.

\section{Programa intervención psicoeducativa y C-Car ${ }^{(38,31)}$}

Este programa está basado en el modelo clínico de la atención de Sohlberg y Mateer ${ }^{(42)}$, que está constituido por etapas. La primera de ella es una intervención psicoeducativa que consta de 6 sesiones individuales semanales de dos horas de duración cada una (ver tabla 3 ), mientras que la segunda parte es un programa de simulación de conducción, en una plataforma similar a los video-juegos y el individuo tiene que procesar y reaccionar ante las señales de tráfico, órdenes verbales, señales de otros automóviles, así como atender a los paisajes diferentes.

Cada sesión que dirige un neuropsicólogo clínico se estructura de la siguiente forma: apartado de psicoeducación de cada dominio cognitivo específico, repercusiones de los déficits del mismo, estrategias de recuperación y su aplicación a la actividad diaria. Se facilita el material a los pacientes y se les solicita que lea de forma anticipada a cada sesión para que el contenido se trabaje de forma individual. Posteriormente se realizan ejercicios mediante los cuales se aprende a aplicar las estrategias, previamente enseñadas, y 


\section{Tabla 2. Descripción del programa ATP}

\begin{tabular}{|c|c|}
\hline Nivel atencional & Tipo de tarea utilizada en la intervención \\
\hline $\begin{array}{l}\text { Atención focalizada y } \\
\text { sostenida }\end{array}$ & $\begin{array}{l}\text { - Tachar un objetivo en tareas de cancelación de figuras y números. } \\
\text { - } \quad \text { Cintas de atención: series de números presentados auditivamente, que deben } \\
\text { ser identificados dando un golpe en la mesa cuando aparecen. } \\
\text { - } \quad \text { Restar mentalmente números. } \\
\text { - Tareas de la vida diaria: buscar en guías, mapas, entre otros. }\end{array}$ \\
\hline Atención selectiva & $\begin{array}{l}\text { - Tachar un objetivo en tareas de cancelación de figuras y números con estí- } \\
\text { mulos distractores. } \\
\text { - Cintas de atención: series de números presentados auditivamente con fondo } \\
\text { de ruido, que deben ser identificados dando un golpe en la mesa cuando } \\
\text { aparecen. } \\
\text { - Tareas de la vida diaria: buscar en guías, mapas, entre otros mientras que se } \\
\text { escucha música de fondo. }\end{array}$ \\
\hline Atención alternante & $\begin{array}{l}\text { - Tachar un objetivo, que cambia cada quince segundos, en tareas de cance- } \\
\text { lación de figuras y números. } \\
\text { - Alternar sumas y restas, tachar números pares e impares, y tareas tipo Stroop } \\
\text { de tamaño y posición. }\end{array}$ \\
\hline Atención dividida & $\begin{array}{ll}\text { - } & \text { Ejecutar simultáneamente la prueba de cancelación visual y auditiva } \\
\text { - } & \text { Emparejar cartas, se tienen que clasificar de acuerdo a categorías junto con } \\
& \text { otra tarea } \\
\text { - } & \text { Tareas de la vida diaria }\end{array}$ \\
\hline
\end{tabular}

\section{Tabla 3. Programa psicoeducativo específico para pacientes con tumores cerebrales}

\section{\begin{tabular}{|l|l|}
\hline Sesión y dominio & Estructura de la sesión \\
\hline
\end{tabular}}

Sesión 1:

Problemas

cognitivos
- Explicación del significado e implicaciones de la función cognitiva, su relación con el cerebro y la diferencia entre las quejas cognitivas a nivel subjetivo y los déficits cognitivos.

- Revisión de los dos tipos de intervención que se van a utilizar en este programa de rehabilitación.

- Factores (médicos, psicológicos y sociales) que influyen en el funcionamiento cognitivo y discutir las experiencias personales de déficit a nivel individual.

- Estrategias generales que mejoran el rendimiento cognitivo.

- Introducción del programa atencional, impartido por ordenador: C-Car.

- Administrar las tareas inter-sesión: registros de las situaciones de deficit y de los factores que influyen de forma positiva o negativa.

- Discusión de los problemas de la vida diaria asociados las alteraciones de las funciones cognitivas, recogidos en los registros que se entregaron en la sesión 1.

- Introducción de la atención, memoria y funciones ejecutivas y la relación entre ellas.

Sesión 2:

Atención, memoria y funciones ejecutivas
- Revisión de las técnicas de intervención: re-entrenamiento (repetición), compensación de los procesos afectados y uso es dispositivos externos.

- Entrenamiento en relajación progresiva.

- Tareas inter-sesión: identificación de situaciones diarias complicadas y aplicación de las estrategias aprendidas. Continuar con el registro de situaciones problemas y práctica de los ejercicios de relajación. 
Tabla 3. Programa psicoeducativo específico para pacientes con tumores cerebrales (continuación)

\begin{tabular}{|c|c|}
\hline Sesión y dominio & Estructura de la sesión \\
\hline $\begin{array}{l}\text { Sesión 3: } \\
\text { Atención }\end{array}$ & $\begin{array}{l}\text { - Repaso de los ejercicios realizados entre las sesiones y la puesta en marcha } \\
\text { de las estrategias en las situaciones diarias. } \\
\text { - } \quad \text { Explicación de la implicación de la atención en el funcionamiento diario y } \\
\text { de las consecuencias de los problemas de la atención en las diferentes áreas } \\
\text { de la vida de una persona. } \\
\text { - Diferenciación de los diferentes niveles atencionales. } \\
\text { - } \\
\text { Identificación de factores psicológicos que influyen en la atención: interés, } \\
\text { motivación, confianza en si mismo. } \\
\text { - Aportar las estrategias específicas para su mejora: prevenir la distracción. } \\
\text { - Tareas entre sesiones: descripción y registro de situaciones complicadas e } \\
\text { identificación de estrategias aplicadas. }\end{array}$ \\
\hline
\end{tabular}

Sesión 4:

Funciones ejecutivas: planificación y auto-regulación

- Repaso de los ejercicios realizados entre las sesiones y la puesta en marcha de las estrategias en las situaciones diarias.

- Introducción sobre los procesos de planificación y auto-regulación, implicación en el funcionamiento diario y las consecuencias de presentar problemas en estas funciones.

- Utilización del programa de planificación: "Siete pasos" para la planificación de una sola actividad y evitar la presión del tiempo. Se eligen dos tareas y se práctica la puesta en marcha de este programa que incluye la realización de un plan de trabajo.

- Repaso de los ejercicios realizados entre las sesiones y del programa de planificación.

- Discusión sobre las tres etapas de la memoria: codificación, almacenamiento y recuperación, y cómo las alteraciones en los procesos nmésicos afectan a la vida diaria.

Sesión 5:

- Información: memoria de trabajo, memoria a corto y largo plazo.

Memoria

- Estrategias específicas que mejoran el rendimiento cognitivo: técnicas de mnemotécnica, señales que facilitan la recuperación de la información, repetición y generación de rutinas.

- Aprendizaje del uso de dispositivos externos: listas de elementos, uso de agendas y diarios y/o aparatos electrónicos.

- Tareas entre sesiones: descripción y registro de situaciones complicadas e identificación de estrategias aplicadas.

Sesión 6:

Resumen

- Repaso de los ejercicios realizados entre las sesiones, principalmente de la memoria

- Resumen de las sesiones anteriores: recapitulación de los ejercicios realizados y estrategias aprendidas.

- Énfasis en el uso de las estrategias y cómo solventar los problemas en la vida diaria, mejorando la eficacia funcional.

- Práctica del programa de los siete pasos.

- Repaso de ejercicios que mejoren la lectura. 
las ayudas específicas en las situaciones específicas e individuales de la vida diaria. Las tareas entre sesiones que se disponen están incluidas en el libro de texto y son revisadas por el terapeuta de forma previa a cada sesión individual, de esta forma, los autores consideran que cada tema específico es trabajado en tres momentos diferentes. Por su parte, los ejercicios básicos del programa de atención C-Car incluyen:

1. Formación de palabras que aparecen de forma visual. Se presentan dos letras que pueden combinarse con múltiples alternativas y el individuo tiene que elegir si la combinación de dos conjuntos de letras forma una palabra.

2. Contar cartas que se presentan de forma auditiva. Cada carta contiene diferentes palabras (ciudades, países y ríos) y después se presenta un dígito $(4,5,6)$ : el sujeto tiene que decidir si el número de letras de la palabra coincide con el dígito que acababan de presentar.

3. Aritmética. En las señales de tráfico de la plataforma se plantean operaciones matemáticas: sumas y restas, de diferente complejidad y el paciente debe decidir si el resultado es el correcto.

4. Alfabeto. De forma auditiva se le presentan diferentes destinos y se le pide que preste atención a la primera letra de cada destino, solicitándole que indique si cada dos destinos las letras iniciales se muestran o no en orden alfabético.

\section{OTRAS INTERVENCIONES DEL DETERIORO COGNITIVO EN PACIENTES CON TUMORES CEREBRALES}

Además de los programas de rehabilitación neuropsicológica detallados, se existen dos tipos de acciones, en fase experimental, que tiene como objetivo prevenir o disminuir el deterioro cognitivo asociado al tumor o a los tratamientos oncológicos. En primer lugar, aquellas in- tervenciones realizadas durante la planificación de los tratamientos oncológicos para evitar la aparición de déficits en determinadas funciones cognitivas. Uno de los estudios más relevante es el que se está realizando por el Radiation Therapy Oncology Group (RTOG 0933), que tiene como objetivo proteger las estructuras implicadas en procesos de aprendizaje y memoria verbal durante la aplicación de la radioterapia holocraneal en pacientes con metástasis cerebrales, sin comprometer la eficacia terapéutica de esta técnica. Diferentes autores ${ }^{(51,52)}$ han postulado que el hipocampo, el circuito límbico y regiones neuronales de células madres (neural stem cell) desempeñan un papel determinante en el rendimiento de procesos mnésicos y atencionales, así como en tareas específicas de las funciones ejecutivas. Además, se considera que la exclusión de las regiones neuronales durante la administración de la radioterapia holocraneal, podría permitir que las zonas subgranurales del hipocampo puedan realizar las tareas de reparación del daño celular en diferentes áreas de córtex cerebral, tras la aplicación de la radiación.

En segundo lugar, están los tratamientos farmacológicos utilizados para la prevención y control del deterioro cognitivo en pacientes con tumores cerebrales. Entre ellos los más relevantes son ${ }^{(6)}$ :

- Memantina: antagonista de los receptores de la NMDA, cuyo papel ha sido ampliamente analizado en la Enfermedad de Alzheimer. En pacientes con tumores cerebrales no hay datos concluyentes sobre su efecto en la prevención de los déficits cognitivos.

- Metilfenidato: fármaco que bloquea la recaptación de noradrenalina y dopamina en las neuronas presinápticas y aumenta la liberación de estas monoaminas al espacio extra-neuronal. Inicialmente se planteó que la aplicación de este fármaco se puede asociar con mejor ren- 
dimiento en velocidad de procesamiento de la información, memoria y funciones ejecutivas; sin embargo en pacientes con neoplasias cerebrales únicamente se observó un patrón de mejoría en el primer proceso cognitivo(53).

- Modfinilo: sustancia encargada de potenciar la actividad alfa-adrenérgica localizada de forma específica en el cerebro. Los estudios hallados hasta la fecha no arrojan información sobre una mejoría en los procesos atencionales tras su aplicación para pacientes con tumores cerebra$\operatorname{les}^{(53)}$.

- Lotironina: fármaco utilizado en el tratamiento de mantenimiento en pacientes con hipotiroidismo que se asocia con una mejoría en el eje hipotálamicopituitario. Wefel et al. ${ }^{(54)}$ observaron una mejoría sólo en la función psicomotriz tras la aplicación de esta sustancia, en una muestra de pacientes con tumores cerebrales que habían recibido radioterapia previamente.

- Donepezilo: inhibidor específico y reversible de la acetilcolinesterasa predominante en el cerebro. Se ha mostrado como un fármaco eficaz para la disminución de síntomas cognitivos, la fatiga y la sensación de confusión en pacientes con Enfermedad de Alzheimer y Parkinson. En pacientes con gliomas de bajo grado que habían recibido radioterapia se evidenció una disminución de los déficits clínicos en atención y memoria, tras la utilización de este fármaco durante 24 semanas; además los pacientes indicaban una mejoría en el estado de ánimo y en la calidad de vida.

\section{CONCLUSIONES}

Un importante número de pacientes con tumores cerebrales puede presentar déficits en algunas funciones cognitivas, principalmente atención, memoria y funciones ejecutivas. A pesar de la naturaleza leve o moderada de los mismos, se ha puesto de manifiesto su influencia negativa en la calidad de vida de los pacientes así como en la reincorporación de aquellos con mejor pronóstico a las diferencias facetas de la vida.

Por ello en los últimos años se están desarrollando tratamientos de rehabilitación, tanto farmacológicos como neuropsicológicos que ayudan a disminuir las alteraciones cognitivas o prevenir su aparición. Respecto al primer tipo, los tratamientos se encuentran en fase experimental y no existe evidencia científica sobre el efecto de los mismos en el rendimiento de algunas funciones cognitivas, principalmente en la memoria. En relación a la Rehabilitación Neuropsicológica, los ensayos clínicos publicados muestran la eficacia de estos programas en la disminución de los déficits de atención, memoria y algunos procesos ejecutivos en pacientes con tumores cerebrales. En este sentido, los autores han recomendado que los programas de rehabilitación incluyan estrategias específicas para los diferentes niveles atencionales, y la mayor parte de los estudios revisados están basados en el modelo teórico y el tratamiento propuesto por Sohlberg y Mateer ${ }^{(41,42)}$. Asimismo, las estrategias para los procesos ejecutivos (planificación y organización) aplicadas en los estudios revisados, forman parte de programas específicos de rehabilitación de funciones ejecutivas, que han mostrado su eficacia en pacientes con daño cerebral adquirido(44,46). Por último, la rehabilitación de la memoria ha sido otro de los objetivos perseguidos en las intervenciones neuropsicológicas revisadas, con resultados de mejoría en el funcionamiento de la memoria verbal $(35,38-40)$.

Adicionalmente, la mayor parte de autores han coincidido en destacar que los programas de intervención neuropsicológica en pacientes con tumores cerebrales deben incluir: un módulo inicial psicoedu- 
cativo sobre los déficits cognitivos y su interferencia en la vida diaria, así como un entrenamiento en estrategias de auto-regulación emocional y técnicas cognitivasconductuales en los programas de rehabilitación, que ayuden a afrontar y adaptarse a la enfermedad oncológica ${ }^{(6,33-35)}$. Además, su inclusión facilitará el afrontamiento de los déficits, mantenimiento de la atención y el manejo de las emociones negativas asociadas. Así, el marco de trabajo que suscribe este tipo de tratamiento puede unir el rendimiento de las tareas entrenadas en los programas de rehabilitación neuropsicológica y el funcionamiento de las actividades de la vida diaria ${ }^{(33)}$.

A pesar de los datos que respaldan la viabilidad de los programas de Rehabilitación Neuropsicológica en pacientes con tumores cerebrales ${ }^{(35)}$, no existe acuerdo respecto al momento más idóneo para su aplicación. Este hecho se debe principalmente a que los déficits cognitivos en esta población no se asocian únicamente al tumor, sino también a los tratamientos oncológicos. Existen dos propuestas:

- Intervención inmediata: el programa de rehabilitación se aplica después del diagnóstico y exéresis de la lesión, de acuerdo al cuarto principio de los programas de Rehabilitación Neuropsicológica, que indicaba la necesidad de iniciar dicha intervención en las fases iniciales del daño cerebral.

- Intervención diferida: el programa de rehabilitación se realiza de forma posterior a la aplicación de los tratamientos oncológicos adyuvantes, habiendo transcurrido el tiempo suficiente para la aparición de los déficits cognitivos asociados a estos tratamientos.

Una alternativa a ambas opciones es la propuesta por Locke et al. ${ }^{(34)}$ que supone realizar la intervención neuropsicológica de forma ambulante, al mismo tiempo que se aplica la Radioterapia en aquellos pacientes que sea necesario. De esta forma, se podría facilitar la adherencia a los programas de intervención, disminuyendo el coste personal y material que supone para algunos enfermos acudir varias veces en la misma semana a los centros de Rehabilitación; así como potenciar la conciencia de los déficits y sus implicaciones en estos desde los momentos iniciales de la enfermedad. Por su parte, el programa desarroIlado por Gehring et al., (31,38) también intenta potenciar la adherencia a la intervención en pacientes con gliomas, al diseñar un software que permite el entrenamiento de procesos atencionales en el domicilio y así poder utilizarlo en cualquier momento de la enfermedad y, que a su vez permite la generalización de los resultados a la vida diaria del paciente.

Respecto a las limitaciones metodológicas, la falta de seguimientos superiores a seis meses después de la intervención es la más frecuente en los estudios revisados, que sólo aporta información sobre el efecto inmediato de los programas de rehabilitación. Gehring et al.(6) indicaron la necesidad de mayores seguimientos en este tipo de pacientes, ya que puede requerirse un tiempo superior a tres o seis meses para integrar las estrategias aprendidas en la vida diaria y en el nivel funcional de estos. Otra de las limitaciones hace referencia al escaso tamaño muestral de las intervenciones que ha provocado incluso que en algunos estudios no se hayan podido realizar análisis estadísticos que valorasen el cambio después de la intervención. En relación a la muestra, la diversidad de los tumores cerebrales puede afectar a la generalización de los resultados, ya que cada tipo de tumor tiene unas características clínicas y tratamientos médicos específicos, y por lo tanto causar unos déficits cognitivos y emocionales que determinen el tipo de intervención neuropsicológica. En último lugar, algunos autores han indicado la dificultad para determinar el éxito de los programas de rehabilitación debido 
a la interferencia de múltiples factores, entre ellos la aplicación de los mismos instrumentos de evaluación neuropsicológica en diferentes momentos de medida para definir el efecto de dichas intervenciones. Por este motivo, en los últimos se está utilizando un índice de cambio estadístico y clínico que supone una medida rigurosa de las variaciones observadas en cada paciente, no son atribuibles a los errores de la medida o a los efectos de la práctica ${ }^{(55)}$.

\section{REFERENCIAS BIBLIOGRÁFICAS}

1. Peterson K. Brain tumors. Neurol Clin 2001;19:887-90. Doi:10.1016/S07338619(05)70052-9

2. Ferlay J, Parkin DM, Steliarova-Foucher E. Estimates of cancer incidence and mortality in Europe in 2008. Eur J Cancer 2010;46:765-81. Doi: 10.1016/j. ejca.2009.12.014.

3. Fox SW, Lyons D, Farace E. Symptom clusters in patients with high-grade glioma. J Nurs Scholarsh 2007;39:61-7. Doi: 10.1111/j.1547-5069.2007.00144.x

4. Giovagnoli A R, Colombo E, Boiardi A. Facets and determinants of quality of life in patients with recurrent high grade glioma. J Neurol Neurosurg Psychiatr 2005;76:5628. Doi:10.1136/jnnp.2004.036186

5. Li J, Bentzen SM, Li J, Renschler M, Mehta MP. Relationship between neurocognitive function and quality of life after whole-brain radiotherapy in patients with brain metastases. Int J Radiat Oncol Biol Phys 2008;71:64-70. Doi: 10.1016/j. ijrobp.2007.09.059

6. Gehring K, Aaronson, Gundy CM, Taphoorn M, Sitskoorn MM. Predictors of Neuropsychological Improvement Following Cognitive Rehabilitation in Patients with Gliomas." J Inter Neuropsychol Soc 2010;17:256-66. Doi: 10.1017/ S1355617710001530

7. Ek L, Almkvist O, Wiberg MK, Stragliotto G, Smits A. Early cognitive impairment in a subset of patients with presumed lowgrade glioma. Neurocase 2010;16:503-11. Doi: 10.1080/13554791003730634

8. Giovagnoli A.Investigation of cognitive impairments in people with brain tumors." J Neurooncology 2012;108:277-83. Doi: 10.1007/s11060-012-0815-6.

9. Hottinger AF, Yoon $H$, Deangelis LM, Abrey LE. Neurological outcome of longterm glioblastoma survivors. J Neurooncol 2009;95:301-5. Doi: 10.1007/s11060009-9946-9

10. Klein M, Taphoorn MJ, Heimans JJ, van der Ploeg HM, Vandertop WP, Smit EF. Neurobehavioural status and health-related quality of life in newly diagnosed high-grade glioma patients. J Clin Oncol 2001;19:4037-47.

11. Klein M EN, van der Ploeg HM, KasteleijnNolst Trenite DG, Aaronson NK, Taphoorn MJ et al. Epilepsy in low-grade gliomas: the impact on cognitive function and quality of life. Ann Neurol 2003;54:514-20. Doi: 10.1002/ana.10712

12. Imperato JP, Paleologos NA, Vick NA. Effects of treatment on long-term survivors with malignant astrocytomas. Ann Neurol 1990;28:818-22. Doi: 10.1002/ ana.410280614

13. Laack NN, Brown PD, Ivnik RJ, Furth AF, Ballman KV, Hammack JE, et al. Cognitive function after radiotherapy for supratentorial low-grade glioma: A North Central Cancer Treatment Group prospective study. Int J Radiat Oncol Biol Phys 2005;63:117583. Doi: 10.1016/j.ijrobp.2005.04.016

14. Meyers CA, Hess KR, Yung WK, Levin VA. Cognitive function as a predictor of survival in patients with recurrent malignant glioma. J Clin Oncol 2000;18:646-50.

15. Taphoorn M. Neurocognitive sequelae in the treatment of low-grade gliomas. Semin Oncol 2003;30(6 Suppl 19):45-8. Doi: 10.1053/j.seminoncol.2003.11.023

16. Taphoorn M, Klein HM. Cognitive deficits in adult patients with brain tumors. Lancet Neurol 2004;3:159-68. 
17. Tucha OS, Preier M, Lange K. Cognitive deficits before treatment among patients with brain tumors. Neurosurgery 2000;47:324-34.

18. Douw LK, Fagel SA, Van den Heuvel J, Taphoorn M, Aaronson NK et al. Cognitive and radiological effects of radiotherapy in patients with low-grade glioma: long term follow up. Lancet Neurol 2009 8:810-18. Doi: 10.1016/S1474-4422(09)70204-2.

19. Heimans JJ, Reijneveld J. Factors affecting the cerebral network in brain tumor patients. J Neurooncol 2012;108:231-7. Doi: 10.1007/s11060-012-0814-7.

20. Henriksson R, Asklund T, Poulsen HS. Impact of therapy on quality of life, neurocognitive function and their correlates in glioblastoma multiforme: A review. J Neurooncol 2011;104:639-46. Doi:10.1007/ s11060-011-0565-x

21. Klein $M$, Aaronson NK, Van der Ploeg, HM, Grit J, Muller M, et al. Effect of radiotherapy and other treatment related factors on mild term to long term cognitive squeals in low grade gliomas: A comparative study. Lancet 2002;360:1361-8.

22. Sanz A, Olivares ME, Barcia JA. Efectos cognitivos de la radioterapia en gliomas de bajo grado. Psicooncología 2011;8:23154. Doi: 10.5209/rev_PSIC.2011.v8.n23.37879

23. Scoccianti S, Detti B, Cipressi S, Iannalfi A, Franzese C, Biti G. Changes in neurocognitive functioning and quality of life in adult patients with brain tumors treated with radiotherapy. J Neurooncol 2012;108:291-308. Doi: 10.1007/s11060012-0821-8

24. Meyers CA, Weitzner MA, Valentine AD, Levin VA. Methylphenidate therapy improves cognition, mood, and function of brain tumor patients. J Clin Oncol 1998;16:2522-7.

25. Langenbahn DM, Ashman T, Cantor J, Trott C. An evidence-based review of cognitive rehabilitation in medical conditions affecting cognitive function. Arch Physl Med
Rehabil 2013;94:271-86. Doi: 10.1016/j. apmr.2012.09.011

26. Cicerone KD, Langenbahn DM, Braden C, Malec JF, Kalmar K, Fraas M, et al. Evidence-based cognitive rehabilitation: Updated review of the literature from 2003 through 2008. Arch Phys Med Rehabil 2011;92:519-30. Doi: 10.1016/j. apmr.2010.11.015.

27. Paúl-Lapiedra N, Bilbao-Bilbao A, RíosLago M. Rehabilitación Neuropsicológica. En: Tirapu J, Ríos-Lago M, Maestú F. Manual de Neuropsicología. 2a ed. Barcelona: Viguera, 2011.p 495-518.

28. Muñoz-Céspedes JM, Tirapu J. Rehabilitación Neuropsicológica. Madrid: Editorial Síntesis, 2001.

29. Abrey LE. The impact of chemotherapy on cognitive outcomes in adults with primary brain tumors. J Neurooncol 2012;108:28590. Doi: 10.1007/s11060-012-0807-6

30. Zangwill OL. Psychological aspects of rehabilitation in cases of brain injury. $\mathrm{Br}$ J Psychol 1947, 37:60-69. Doi: 10.1111/ j.2044-8295.1947.tb01121.x

31. Gehring K, Aaronson N, Taphoorn M, Sitskoorn M. A description of a cognitive rehabilitation programme evaluated in brain tumour patients with mild to moderate cognitive deficits. Clin Rehabil 2011;25:67592. Doi: 10.1177/0269215510395791.

32. Wilson BA. Memory rehabilitation in brain-injured people. In: Stuss DT, Winocur G, Robertson IH, editors. Cognitive Neurorehabilitation. New York: Cambridge University Press; 2005.p 333-46.

33. Brigidi B, Friedman H, Raynor R. Cognitive rehabilitation in patients with primary malignant brain tumors: A case study series using an acceptance-based cognitive training approach $(\mathrm{N}=10)$. Abstract QL-19. Presentation for the 2007 Twelfth Annual Meeting of the Society for NeuroOncology (SNO). Neuro-Onc 2007;9: 568.

34. Locke DE, Cerhan JH, Wu W, Malec JF, Clark MM, Rummans TA, Brown PD. Cog- 
nitive rehabilitation and problem-solving to improve quality of life of patients with primary brain tumors: A pilot study. J Support Oncol 2008;6:383-91.

35. Hassler MR, Elandt K, Preusser M, Lehrner J, Binder P, Dieckmann K, Rottenfusser A, Marosi C. Neurocognitive training in patients with high-grade glioma: A pilot study. J Neurooncol 2010;97:109-15. Doi: 10.1007/s11060-009-0006-2.

36. Sherer M, Meyers CA, Bergloff P. Efficacy of postacute brain injury rehabilitation for patients with primary malignant brain tumors. Cancer 1997;80:250-7.

37. Duval J, Coyette F, Seron X. Rehabilitation of the central executive component of working memory: A re-organisation approach applied to a single case. Neuropsychol Rehabil 2008;18:430-60. Doi: 10.1080/09602010701573950.

38. Gehring K, Sitskoorn MM, Gundy CM, Sikkes SA, Klein M, Postuma TJ, et al. Cognitive rehabilitation in patients with gliomas: A randomized, controlled trial. J Clin Oncol 2009;27:3712-22.

39. Metzler-Baddeley C, Jones RW. Brief communication: cognitive rehabilitation of executive functioning in a case of craniopharyngioma. Appl Neuropsychol 2010;17:299304. Doi: 10.1080/09084282.2010.523394.

40. Zucchella C, Bartolo M, Di Lorenzo C, Villani V, Pace A. Cognitive impairment in primary brain tumors outpatients: A prospective cross-sectional survey. J Neurooncol 2013;112:455-60. Doi: 10.1007/ s11060-013-1076-8

41. Sohlberg MM, Mateer CA.Effectiveness of an attention-training program. J Clin Exp Neuropsychol 1987; 9: 117-130.

42. Sohlberg MM, Mateer CA. Attention Process Training (APT). Puyallup, WA: Association for Neuropsychological Research and Development; 1986.

43. Teixidor P, Gatignol P, Leroy M, Masuet-Aumatell C, Capelle L, Duffau H. Assessment of verbal working memory before and after surgery for low-grade glioma. J Neuroon- col 2007;81:305-13. Doi: 10.1007/s11060006-9233-y

44. Levine B, Robertson IH, Clare L, Carter G, Hong J, Wilson BA, et al. Rehabilitation of executive functioning: An experimental-clinical validation of goal management training. J Int Neuropsychol Soc 2000;6:299-312.

45. Duncan J, Emslie H, Williams P, Johnson $\mathrm{R}$, Freer $\mathrm{C}$. Intelligence and the frontal lobe: the organization of goal-directed behavior. Cogn. Psychol 1996;30:257-303.

46. Winkens I, Van Heugten C, Wade DT, Habetsa EJ, Fasotti L- Efficacy of time pressure management in stroke patients with slowed information processing: A randomized controlled trial. Arch Phys Med Rehabil. 2009;90:1672-9. Doi: 10.1016/j. apmr.2009.04.016.

47. Chu BC, Millis S, Arango-Lasprilla JC, Hanks $R$, Novack T, Hart Measuring recovery in new learning and memory following traumatic brain injury: A mixed-effects modeling approach. J Clin Exp Neuropsychol 2007;29:617-25. Doi: 10.1080/13803390600878893

48. Sherer M, Stouter J, Hart T, Nakase-Richardson R, Olivier J, Manning E, Yablon, S.A. Computed tomography findings and early cognitive outcome after traumatic brain injury. Brain Inj 2006;20:997-1005.

49. Stern Y. Cognitive reserve. Neuropsychologia. 2009;47: 2015-28. Doi:10.1016/j. neuropsychologia.2009.03.004

50. Bernabeu-Verdúa J, López-Luengo B, Fournier-del Castillo C, Cañete-Nieto, A, Suárez Rodríguez J, Castel Sánchez V. Aplicación del Attention Process Training dentro de un proyecto de intervención en procesos atencionales en niños con cáncer. Rev Neurol 2004;38:482-6.

51. Gondi V, Toméa WA, Mehtaa M. Why avoid the hippocampus? A comprehensive review. Radiother Oncol 2010:97:370-6. Doi: 10.1016/j.radonc.2010.09.013.

52. Marsh JC, Gielda BT, Herskovic AM, Abrams RA. Cognitive sparing during the administration of whole brain radiotherapy 
and prophylactic cranial irradiation: Current concepts and approaches. J Oncol 2010; Doi:10.1155/2010/198208.

53. Patwardhan SY, Myszka KA, Collins R, Etzel CJ, Meyers CA, Wefel JS. Assessment of the efficacy of immediate release methylphenidate, sustained release methylphenidate, and modafinil for patients with primary brain tumor. J Int Neuropsychol Soc 2010; 16 (Suppl. 1):88.
54. Wefel JS, Patwardhan S, Levin VA. Neurocognitive outcomes associated with combined levothyroxine/liothyronine supplementation in hypothyroid patients with primary brain tumors. Neurooncol 2009;11:642.

55. Maassen $\mathrm{GH}$, Bossema E, Brand N. Reliable change and practice effects: Outcomes of various indices compared. J Clin Exp Neuropsychol 2009;31:339-52. Doi: 10.1080/13803390802169059 
